\title{
Polypose adénomateuse familiale et gène APC
}

Des altérations génétiques perturbant le contrôle de la croissance cellulaire sont à l'origine de la maladie cancéreuse. Certaines de ces modifications génétiques sont transmissibles héréditairement. En particulier, des études épidémiologiques suggèrent qu'environ $15 \%$ des cancers colorectaux sont familiaux. Deux formes héréditaires sont bien caractérisées d'un point de vue clinique : la polypose rectocolique familiale ou polypose adénomateuse familiale (familial adenomatous polyposis), et les cancers colorectaux survenant chez des sujets non atteints de polypose héréditaire (syndrome de Lynch) $\left(m / s n^{\circ} 8-9\right.$, vol. 9, p. 990 et $n^{\circ} 2$, vol. 10, p. 228).

La polypose rectocolique familiale est une maladie à transmission autosomique dominante relativement fréquente (1 pour 10000 naissances), qui se caractérise par l'apparition de centaines de polypes adénomateux dans le côlon et le rectum pendant les premières décennies de la vie. Faute d'ablation chirurgicale, les individus affectés développent un carcinome avant l'âge de 40 ans. A ces polypes peuvent venir s'associer des tumeurs desmoïdes, des tumeurs duodénales, des tumeurs de la vésicule ou des voies biliaires, des ostéosarcomes, des tumeurs de la thyroïde (syndrome de Gardner), ou des tumeurs du système nerveux central (syndrome de Turcot) [1, 2]. L'analyse par cytogénétique puis des études de liaison du génome des membres de plusieurs familles à l'aide de marqueurs génétiques ont permis de démontrer en 1987 que le gène responsable de la polypose adénomateuse familiale est localisé au niveau de la région 5q21-q22 [1]. Structure du gène $A P C$ - fonction de
la protéine

Le gène $A P C$ (adenomatous polyposis coli) a été isolé en $1991\left(\mathrm{~m} / \mathrm{s} n^{\circ} 7, \mathrm{vol}\right.$. 7, p. 718) [3-6]. C'est un long gène qui s'étend sur $300 \mathrm{~kb}$. Il comporte 15 exons de taille inégale (figure $1 A$ ). Une partie de l'exon 9 (nucléotides 934-1236) peut être éliminée par épissage alternatif [3]. La région codante du plus grand transcrit comporte 8535 nucléotides [3]. L'expression de ce gène a pu être mise en évidence dans les lymphocytes et dans de nombreux tissus normaux [3]. Il est également fortement exprimé au cours du développement du système nerveux central [7].

Les formes de maturation différente codent pour des protéines de 2743 et 2844 acides aminés, correspondant à des protéines de masse théorique 300,5 et $311,7 \mathrm{kDa}$, respectivement. De nombreux sites potentiels de glycosylation sont présents, mais la taille observée [8] indique que peu d'entre eux sont utilisés. Des sites potentiels de phosphorylation et myristoylation sont également présents [3], mais il n'est pas établi s'ils sont utilisés, ni s'ils jouent un rôle. Trois régions ont pu être définies d'après la structure primaire de la protéine APC déduite de la séquence nucléotidique (figure 1B). La partie N-terminale ( 900 résidus) est dépourvue de proline et possède onze groupes de répétition en tandem d'un motif dégénéré de 7 acides aminés (acide aminé hydrophobe - X - X - acide aminé hydrophobe - X - X - X), caractéristiques de structures de type hélicoïdal (coiled-coil). La mise en évidence de ces structures a permis de suggérer une possible homodimérisa- tion et/ou une hétérodimérisation avec d'autres facteurs cellulaires $(f i$ gure $1 B$ ). Il a pu être démontré expérimentalement par la suite que cette région est effectivement à l'origine de l'homodimérisation de la protéine $[9,10]$. Un fragment comportant les 55 acides aminés amino-terminaux suffit à former un dimère stable [9] . La région centrale de la protéine APC présente 7 répétitions d'un motif d'une vingtaine d'acides aminés [3]. L'immunoprécipitation de la protéine APC entraîne la copurification de protéines identifiées à l' $\alpha$-caténine et à la $\beta$-caténine $[11,12]$. C'est cette région centrale de la protéine APC qui est responsable de cette interaction (figure 1B). Su et al. ont pu montrer qu'un fragment de 27 acides aminés (résidus 1013 à 1039) est suffisant pour que l'interaction puisse avoir lieu [12]. Enfin, il a été récemment démontré que la protéine APC, localisée dans le cytoplasme de cellules de colonocytes normaux [8], est associée à la tubuline, et que cette interaction induit l'assemblage de ces filaments in vitro [13]. L'extrémité carboxy-terminale est impliquée dans cette interaction. Ce domaine a pour particularité de contenir une région riche en acides aminés basiques (acides aminés 2200-2400) (figure $1 B$ ). L'importance de cette région n'a toutefois pas encore été élucidée.

La $\beta$-caténine est associée avec l'extrémité cytoplasmique de la cadhérine $\mathrm{E}$, molécule d'adhérence (également appelée uvomoruline) importante dans la morphogenèse. L'interaction $\mathrm{E}$ cadhérine-caténine serait essentielle dans le fonctionnement de la cadhérine pour maintenir l'adhérence des cellules épithéliales, vrai- 
semblablement par l'ancrage des cadhérines au cytosquelette. Il est probable que la protéine APC module l'interaction entre cadhérines et caténines, altérant ainsi les mécanismes par lesquels les interactions intercellulaires contrôlent croissance et différenciation cellulaires. La protéine APC pourrait, par exemple, en modulant l'adhérence cellulaire, faciliter le relargage des cellules épithéliales dans la lumière de l'intestin, prévenant ainsi la rétention des cellules qui prolifèrent au niveau des cryptes [14].

\section{Altérations du gène $A P C$}

De nombreuses formes mutées de ce gène ont été retrouvées dans la lignée germinale de patients atteints de polypose familiale [3, 6, 15-17]. Ce sont des mutations ponctuelles (portant essentiellement sur des cytosines), des délétions courtes (1-14 nucléotides), ou des insertions (1-2 nucléotides). Ces altérations intéressent particulièrement l'extrémité 5 ' de la région codante du gène $A P C$ (figure 1C), environ $70 \%$ étant situées dans la partie 5' de l'exon 15 (codons 713-1597) [18]. Les deux délétions les plus fréquemment rencontrées concernent 5 bases (AAAAG) au niveau du codon 1309 (20\% des cas), et une autre séquence de 5 bases (ACAAA) au niveau du codon 1061 (10\% des cas). Toutes ces modifications engendrent directement des codons stop, ou sont à l'origine d'un décalage du cadre de lecture permettant la formation d'un codon stop immédiatement en aval de cette altération. Ces codons stop conduisent à l'expression d'une protéine APC tronquée [8], peu ou pas fonctionnelle. Elle est, cependant, capable de s'associer à la protéine normale [10] et pourrait ainsi l'inactiver, et exercer une activité négative dominante. Seules les formes mutantes de la protéine contenant le domaine de fixation correspondant sont capables de s'associer aux caténines [11, 12]. Enfin, ces protéines tronquées ne peuvent plus s'associer aux microtubules du cytosquelette [19].

Les manifestations ophtalmologiques dépendent étroitement de la position
A - Région codante de l'ARNm codant pour la protéine APC

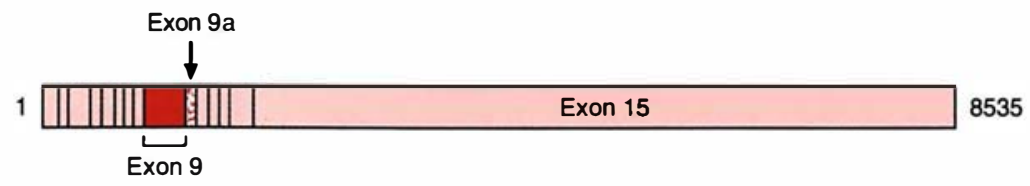

B. Domaines fonctionnels de la protéine APC

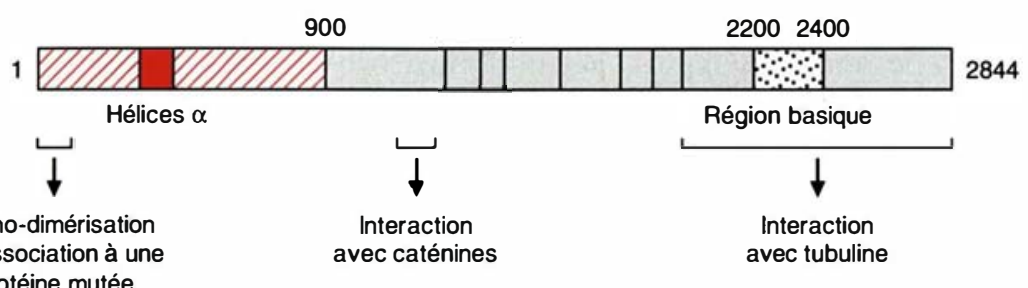

ou association à un protéine mutée

C Sites préférentiels de mutations germinales de la protéine APC

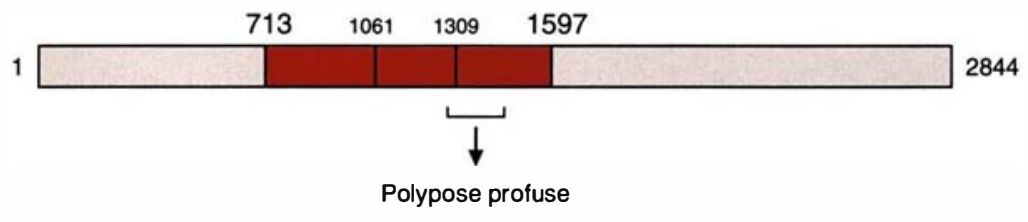

Figure 1. Le gène et la protéine APC. A. Répartition des exons constituant la région codante de l'ARNm codant pour la protéine APC. Une partie de l'exon 9 (nucléotides 934-1236), représentée en rouge, peut être éliminée par épissage alternatif. Le fragment de l'exon 9 restant est appelé exon 9a (pointillé rouge). B. Domaines fonctionnels de la protéine APC. La partie $\mathrm{N}$-terminale (hachurée rouge) possède onze groupes de répétition en tandem d'un motif dégénéré de 7 acides aminés lacide aminé hydrophobe - $X-X$ - acide aminé hydrophobe - $X-X-X)$ permettant $d^{\prime}$ adopter une conformation en hélices $\alpha$. Une partie de la protéine peut être absente (en rouge), en raison de l'épissage alternatif de l'exon 9. Un fragment comportant les 55 acides aminés amino-terminaux suffit à former un dimère stable. La région centrale de la protéine APC présente 7 répétitions d'un motif d'une vingtaine d'acides aminés (barres verticales). Un fragment de 27 acides aminés (résidus 1013 à 1039) est suffisant pour que l'interaction avec les caténines puisse avoir lieu. L'extrémité carboxy-terminale, qui contient une région riche en acides aminés basiques (pointillé noir), permet à la protéine APC de s'associer au cytosquelette. $C$. Sites préférentiels de mutations germinales de la protéine APC. L'intensité de coloration d'une région de la protéine est liée à une fréquence d'observation de mutations plus élevée. Les codons les plus fréquemment altérés (1061 et 1309) sont représentés. La partie de la protéine altérée dans les formes de polypose profuse (1285-1465) est indiquée. 
de l'épithélium de la rétine sont observées uniquement lorsque les altérations se situent après l' exon 9 [20]. Il semble également exister une corrélation entre la localisation de la mutation germinale et le nombre de polypes $[21,22]$ : des mutations affectant les codons 1285 à 1465 paraissent associées à une polypose profuse (plus de 5000 polypes) [15]. A l'inverse, c'est l'extrémité 5 ' du gène $A P C$ qui est altérée dans les formes atténuées (nombre de polypes généralement inférieur à 100) de polypose familiale [23].

En dehors de ces altérations pouvant être transmises génétiquement, des mutations du gène $A P C$ ont également été retrouvées dans la majorité des tumeurs colorectales sporadiques analysées [6, 24, 25]. Là encore, la plupart des mutations sont distribuées dans la première moitié de la protéine, particulièrement au niveau d'une courte région de l'exon 15 correspondant à moins de $10 \%$ de la région codante appelée MCR (mutation cluster region; codons 1286-1513 [15, 26]), et conduisent à l'expression d'une protéine tronquée. Ces mutations ont été détectées dans de petites tumeurs bénignes, suggérant que les mutations du gène $A P C$ seraient à l'origine aussi bien des tumeurs sporadiques que des formes familiales $\left(\mathrm{m} / \mathrm{s} n^{\circ} 11\right.$, vol. $\left.8, \quad p .1000\right)$ [25]. La coopération avec d'autres altérations génétiques conduirait à la formation du carcinome.

\section{Mise en évidence d'altérations}

La mise en évidence directe de mutations germinales peut être envisagée pour dépister des patients à risque avant l'apparition des premiers symptômes. Ces altérations étant germinales, il est possible de les rechercher à partir d'ADN génomique préparé avec des cellules sanguines, facilement accessibles. Certaines mutations du gène $A P C$ peuvent être simplement détectées par électrophorèse d'ADN génomique amplifié, suivi d'une digestion par les enzymes de restriction appropriées ( $R F L P[23$, 27]). Dans d'autres cas, l'analyse du polymorphisme de conformation d'ADN simple-brin (SSCP $[16,23$, 24]), la mise en œuvre de techniques d'électrophorèse sur gel en gradient d'agent dénaturant (DGGE [17]) ou de protection à l'action de la ribonucléase [18] devront être envisagées. Enfin, une technique récemment décrite permet de mettre en évidence une protéine APC tronquée sans séquençage de l'ADN. Des fragments du gène $A P C$ sont amplifiés par PCR, et ces fragments d'ADN sont utilisés dans un test de transcription/traduction in vitro. Une simple électrophorèse suivie d'autoradiographie permet alors de déterminer la taille des protéines synthétisées, et donc de conclure quant à la présence d'une altération conduisant à l'expression d'une protéine tronquée inactive in vivo [28]. La grande majorité des altérations étant portées par l'exon 15 , l'analyse pourra être effectuée en priorité sur cette région du gène $A P C$ [29].

Pour les individus chez lesquels une altération sera trouvée, l'évolution du développement des polypes sera étroitement surveillée par colonoscopie. De même, le développement de tumeurs extracoliques pourra également faire l'objet d'une surveillance particulière. Enfin, ces personnes pourront être psychologiquement préparées à une éventuelle colectomie, et prévenues quant au risque encouru par les générations suivantes.

En revanche, les individus n'ayant pas hérité de l'altération pourront subir un nombre inférieur d'évaluations médicales et de colonoscopies. L'anxiété associée à la présomption de la maladie sera réduite, et les patients pourront être rassurés pour leurs descendants

\section{Marc G. Denis \\ Patrick Lustenberger}

Laboratoire de biochimie spécialisée, institut de biologie, 9, quai Moncousu, 44035 Nantes Cedex, France.

\section{RÉFÉRENCES}

1. Thomas G, Muleris M, Salmon R. La génétique du cancer colorectal. médecine/ sciences $1988 ; 4: 274-80$.

2. Olschwang S, Laurent-Puig P, Thomas G. Expression phénotypique de la polypose adénomateuse familiale. médecine/sciences $1994 ; 10: 454-6$.

3. Groden J, Thliveris A, Samowitz W, Carlson M, Gelbert L, Albertsen $H$, Joslyn G, Stevens J, Spirio L, Robertson M, Sargeant L, Krapcho K, Wolff E, Burt R, Hughes JP, Warrington J, McPherson J, Wasmuth J, Lepaslier D, Abderrahim H, Cohen D, Leppert $M$, White $R$. Identification and characterization of the familial adenomatous polyposis coli gene. Cell $1991 ; 66: 589-600$.

4. Kinzler KW, Nilbert MC, Su LK, Vogelstein B, Bryan TM, Levy DB, Smith KJ, Presinger AC, Hedge $P$, McKechnie D, Finniear R, Markham A, Groffen J, Boguski MS, Altschul SF, Horii A, Ando H, Miyoshi Y, Miki Y, Nishisho I, Nakamura Y. Identification of FAP locus genes from chromosome 5q21. Science 1991 ; 253 : 661-5.

5. Joslyn G, Carlson M, Thliveris A, Albertsen H, Gelbert L, Samowitz W, Groden J, Stevens J, Spirio L, Robertson M, Sargeant L, Krapcho K, Wolff E, Burt R, Hughes JP, Warrington J, McPherson J, Wasmuth J, Lepaslier D, Abderrahim H, Cohen D, Leppert $M$, White $R$. Identification of deletion mutations and three new genes at the familial polyposis locus. Cell 1991; 66:601-23.

6. Nishisho I, Nakamura Y, Miyoshi Y, Miki Y, Ando H, Horii A, Koyama K, Utsunomiya J, Baba S, Hedge P, Markham A, Krush AJ, Petersen G, Hamilton SR, Nilbert MC, Levy DB, Bryan TM, Preisinger AC, Smith KJ, Su LK, Kinzler KW, Vogelstein B. Mutations of chromosome 5q21 genes in FAP and colorectal patients. Science $1991 ; 253$ : 665-9.

7. Bhat RV, Baraban JM, Johnson RC, Eipper BA, Mains RE. High levels of expression of the tumor suppressor gene $A P C$ during development of the rat central nervous system. J Neurosci 1994 ; 14 : 3059-71.

8. Smith KJ, Johnson KA, Bryan TM, Hill DE, Markowitz S, Willson JKV, Paraskeva C, Petersen GM, Hamilton SR, Vogelstein B, Kinzler KW. The $A P C$ gene product in normal and tumor cells. Proc Natl Acad Sci USA $1993 ; 90: 2846-50$.

9. Joslyn G, Richardson DS, White R, Albert $\mathrm{T}$. Dimer formation by an $\mathrm{N}$-terminal coiled coil in the APC protein. Proc Natl Acad Sci USA 1993 ; 90 : 11109-13.

10. Su LK, Johnson KA, Smith KJ, Hill DE, Vogelstein B, Kinzler KW. Association between wild type and mutant $A P C$ gene products. Cancer Res 1993 ; 53 : 2728-31.

11. Rubinfeld B, Souza B, Albert I, Müller O, Chamberlain SH, Masiarz FR, Munemit su $\mathrm{S}$, Polakis $\mathrm{P}$. Association of the APC gene product with $\beta$-catenin. Science $1993 ; 262$ : 1731-4. 
12. Su LK, Vogelstein B, Kinzler KW. Association of the $A P C$ tumor suppressor protein with catenins. Science $1993 ; 262: 1734$ 7.

13. Munemitsu S, Souza B, Müller O, Albert I, Rubinfeld B, Polakis P. The $A P C$ gene product associates with microtubules in vivo and promotes their assembly in vitro. Cancer Res 1994 ; 54 : 3676-81.

14. Hülsken J, Behrens J, Birchmeier W. Tumor-suppressor gene products in cell contacts: the cadherin-APC-armadillo connection. Curr Op Cell Biol 1994 ; 6 : 711-6.

15. Nagase $H$, Nakamura $Y$. Mutations of the APC gene. Hum Mut 1993 ; 2 : 425-34.

16. Mandl M, Paffenholz R, Friedl W, Caspari R, Sengteller M, Propping P. Frequency of common and novel inactivating $A P C$ mutations in 202 families with familial adenomatous polyposis. Hum Mol Genet 1994 ; 3: 181-4.

17. Olschwang S, Laurent-Puig P, Groden J, White R, Thomas $\mathbf{G}$. Germ-line mutations in the first 14 exons of the adenomatous poly posis coli (APC) gene. Am J Hum Genet 1993 ; 52 : 273-9.

18. Mioyshi, $Y$, Ando $H$, Nagase $H$, Nishisho I, Horii A, Miki Y, Mori T, Utsunomiya J, Baba S, Petersen G, Hamilton SR, Kinzler KW, Vogelstein B, Nakamura Y. Germ-line mutations of the $A P C$ gene in 53 familial adenomatous polyposis patients. Proc Natl Acad Sci USA 1992; 89 : 4452-6.
19. Smith KJ, Levy DB, Maupin P, Pollard TD, Vogelstein B, Kinzler KW. Wild-type but not mutant APC associates with the microtubule cytoskeleton. Cancer Res $1994 ; 54$ : $3672-5$.

20. Olschwang $\mathbf{S}$, Tiret A, Laurent-Puig $\mathbf{P}$, Muleris M, Parc R, Thomas G. Restriction of ocular fundus lesions to a specific subgroup of APC mutations in adenomatous polyposis coli patients. Cell 1993 ; 75 : 959-68.

21. Nagase H, Miyoshi Y, Horii A, Aoki T, Ogawa M, Utsunomiya J, Baba S, Sasazuki T, Nakamura $Y$. Correlation between the location of germ-line mutations in the APC gene and the number of colorectal polyps in familial adenomatous polyposis patients. Cancer Res 1992 ; 52 : 4055-7.

22. Nagase H, Miyoshi Y, Horii A, Aoki T. Screening for germ-line mutations in familial adenomatous polyposis patients: 61 new patients and a summary of 150 unrelated patients. Hum Mut 1992 ; 1 : 467-73.

23. Spirio L, Olschwang S, Groden J, Robertson M, Samowitz W, Joslyn G, Gelbert L, Thliveris A, Carlson M, Otterud B, Lynch $\mathrm{H}$, Watson $P$, Lynch $P$, Laurent-Puig P, Burt $R$, Hughes JP, Thomas G, Leppert M, White R. Alleles of the $A P C$ gene: an attenuated form of familial polyposis. Cell $1993 ; 75$ : 951-7.

24. Cottrell S, Bicknell D, Kaklamanis L, Bodmer WF. Molecular analysis of $A P C$ mutations in familial adenomatous polyposis and sporadic colon carcinomas. Lancet $1992 ; 340: 626-30$.
25. Powell SM, Zilz N, Beazer-Barclay $\mathrm{Y}$, Bryan, TM, Hamilton SR, Thibodeau SN, Vogelstein B, Kinzler KW. APC mutations occur early during colorectal tumorigenesis. Nature $1992 ; 359: 235-7$

26. Miyoshi $Y$, Nagase $H$, Ando $H$, Horii A, Ichii S, Nakatsuru S, Aoki T, Miki Y, Mori T, Nakamura Y. Somatic mutations of the $A P C$ gene in colorectal tumors : mutation cluster region in the APC gene. Hum Mol Genet $1992 ; 4: 229-33$.

27. Kraus C, Ballhausen WG. Two intragenic polymorphisms of the APCgene detected by PCR and enzymatic digestion. Hum Genet 1992 ; 88 : 705-6.

28. Powell SM, Petersen GM, Krush AJ, Booker S, Jen J, Giardiello FM, Hamilton SR, Vogelstein B, Kinzler KW. Molecular diagnosis of familial adenomatous polyposis. $N$ Engl J Med 1993 ; 329 : 1982-7.

29. Van Der Luijt R, Khan PM, Vasen $H$, Van Leeuwen $C$, Tops $C$, Roest $P$, Den Dunnen J, Fodde R. Rapid detection of translation-terminating mutations at the adenomatous polyposis coli (APC) gene by direct protein truncation test. Genomics $1994 ; 20$ : $1-4$.

\section{TIRÉS À PART}

M.G. Denis. 